\title{
GEOGRAPHICAL DISTRIBUTION OF MEMBERS
}

\section{UNITED STATES}

\section{Alabama}

Auburn. Doner, Harkin.

BIRMINGHaM. Eagles, W. P. Hammond, Hess, Sewell.

MARION. Brant.

SPRING HiLl. K. Nowlan.

UnIVERSITY. F. A. Lewis, Ott.

\section{Arizona}

Flagstaff. Lampland.

Phoenix. Hannelly.

Tucson. Cresse, Graesser, Leonard.

\section{Arkansas}

ArKadelphia. Garretson.

Clarksville. De Foe.

FAyetTEVILLE. Harding, J.C. Hughes, Nichols, D. P. Richardson, Worth.

LITTLE Rock. Bigbee, d'Unger, C. E. Rose, T. F. Smith.

\section{California}

Atascadero. Schmiedel.

BERKELEY. A. D. B. Andrews, Bernstein, Buck, Cajori, G. C. Edwards, M. W. Haskell, F. Irwin, D. N. Lehmer, Leushner, S. Levy, J. H. McDonald, Noble, T. M. Putnam, Shane, P. Sperry, A. R. Williams, B. C. Wong.

Clanemont. Russell.

Fresno, F. R. Morris.

Hollywood, Entz.

LA Jolla. G. F. McEwen.

Los Angeles. Ames, C. Bell, Boss, Collier, Cooper, Daus, Garver, Glazier, Hann, Havens, E. R. Hedrick, G. H. Hunt, Ickes, G. James, Otis, Sherwood, Showman, Steed, Swenson, E. E. Thomas, Touton, R. A. Wheeler, W. M. Whyburn, Willett, Worthington.

MARE ISLAND. See.

Menlo. V. James.

OAKLAND. Alderton, E. W. McDonald.

ORLAND. Fenner.

Pacific Grove. Barter.

Palo Alto. W. E. Milne.

Pasadena. Basoco, Bateman, E. T. Bell, Birchby, Kennison, Klein, Robertson, E. L. Rose, St. John,
Sturdivant, Tolman, Van Buskirk, M. Ward, Wear, Wolfe, Yost.

Redlands. O. W. Albert.

Salinas. Stager.

SAN DiEgo. Klauber, Küstermann.

SAN Francisco. Hewes, McCarty.

SAN Jost. Heminger.

San Rafael. A. M. Whelan.

SANTA ANA. Whiting.

STANFORD UNIVERSITY. C. L. Alsberg, Blichfeldt, Crum, Durand, R. L. Green, T. J. Hoover, L. M. Hoskins, Hotelling, W. A. Manning, Moreno, D. L. Webster.

Stockton. Corbin, Weiss.

\section{Colorado}

Boulder. De Long, C. A. Hutchinson, Jekel, Karnow, Kempner, Kendall, Lester.

Colorado Springs. Sisam.

Denver. Epsteen, Powers, Remington.

Fort Collins. A. G. Clark.

GoLDEN. Fitterer, Risley.

\section{Connecticut}

BRIDGEPORT. M. G. Haseman.

HARTFORD. Bjorn, A. T. Bunyan, Dadourian, C. H. Davis, Dewey, Dorweiler, Doxsee, Elston, B. D. Flynn, J. D. Flynn, Haradon, W. V. B. Hart, E. C. Henderson, Hezlett, E. B. Hill, Holley, J. E. Hoskins, Kaufman, Keffer, Laird, Larus, E. B. Morris, Paine, Sinnott, Springer, Tarbell, Welch.

Middletown. B. H. Camp, M. C. Foster, Howland.

Milford. J. Rosenbaum, S. B. Rosenbaum.

New Haven. Barney, Benton, E. W. Brown, H. T. Burgess, Engstrom, Fisher, Gehman, Kovarik, Lindsay, Longley, Miles, L. T. Moore, Ore, L. Page, Pierpont, Rawles, C. A. Shook, P. F. Smith, Stoughton, H. Thompson, Tracey, Uhler, Whittemore, W. A. Wilson.

NEw London. Dimick, Leib.

NoRwich. Leavens. 


\section{Delaware}

Newark. G. A. Harter, J. C. Rees.

\section{District of Columbia}

WAshington. O. S. Adams, William James Berry, Breit, Cromwell, H. K. Cummings, Darling, English, Erwin, H. B. Hedrick, Hodgkins, Humphreys, Lambert, Landry, Maddrill, E. C. Phillips, Ramler, J. N. Rice, R. B. Robbins, Roeser, Shenton, Van Orstrand, Wernicke, Woodard, Woolard, Yurow.

\section{FLORIDA}

Gainesville. Dostal, Kusner, Phipps, T. M. Simpson.

Jacksonville. E. A. Porter.

\section{Georgia}

Athens: Barrow, W. S. Beckwith, R. P. Stephens.

Atlanta. C, E. Bennett, Bleckley, Brine, F. Field, Hammel, Morton, M. R. Richardson, Skiles, D. M. Smith.

Decatur. H. A. Robinson.

EMORY UnIVERSITY. Messick.

GaINEsville. Allison.

LaGrange. E. A. Bailey.

MACON. Holder, C. F. Whittaker.

Rome. Hightower.

\section{HaWAII}

HoNOLuLu. Eckles.

\section{IDAHO}

Caldwell. J. M. Rankin.

Moscow. J. R. Bender, E. Taylor.

Pocatello. West.

\section{ILLINOIS}

Bloomington. M. Hunt.

Blufrs. C. C. Carter.

Carthage. Van Velzer.

Charleston. E. H. Taylor.

Chicago. Bamforth, Bardell, Barnard, Bartky, Bibb, G. A. Bliss, Budd, D. F. Campbell, E. R. Carter, H. E. Cobb, Curjel, Dickson, Everett, Feltges, Georges, Granville, L. M. Graves, Griffiths, Harza, Hornberger, Jenkins, B. W. Jones, Kinney, Krathwohl, Kurzin, E. P. Lane, LaPaz, Laves, Levinson, Logsdon, Lunn, W. D. MacMillan, E. H. Moore, W. O. Morris, Morrison, D. C. Morrow, F. R. Moulton, Myers, Oppenheim, Palmer, Paul, Ross, Sanger, Schottenfels, A. R.
Schweitzer, E. O. Schweitzer, R. C. Shook, Slaught, Teach, Van der Vries, Wiggin, Wilczynski, Y. K. Wong, Woolf, J. W. A. Young.

EUREKA. Newson.

Evanston. O. E. Brown, Curtiss, $\mathrm{H}$. L. Garabedian, Griffiths, Holgate, E. J. Moulton, Simmons, Wall, F.E. Wood.

FREEPORT. Mensenkamp.

Galesburg. Sellew, M. E. Taylor.

JoliET. E. L. Thompson.

LAKe Forest. H. B. Curtis, E. L. Milne.

LaSalle. E. H. Carus, M. H. Carus. LEBANON. Stowell.

LISLE. Fleisig.

NoRmal. Mills.

OAK PARK. Escoti.

PEORIA. Comstock.

ROCKFORD. McGavock.

ROCK Island. Cederberg.

URBANA. B. M. Armstrong, H. W. Bailey, R. D. Carmichael, Coble, Crathorne, Emch, C. F. Green, Hazlett, Hoersch, C. Hopkins, Ketchum, H. Levy, Libman, Lytle, Maria, B. I. Miller, G. A. Miller, T. W. Moore, Oakley, Ogg, J. B. Shaw, Steimley, Townsend.

WhEATON. Hillard.

\section{INDIANA}

Bloomington. H. T. Davis, Davisson, Hanna, Hennel, Rothrock, K. P. Williams.

Columbia City. Knisely.

Fort WAyne. F. B. Mead, F. W. Merrill.

INDIANAPOLIS. Beckett, L. C. Cox, E. N. Johnson.

LafayetTe. Copp, P. Cramer, Edington, G. H. Graves, Hodge, W. Marshall, T. E. Mason, R. B. Stone, Westlund, Zehring.

Muncie. P. D. Edwards, Shively.

Notre DaME. Hull.

\section{IowA}

Ames. E. S. Allen, J. T. Colpitts, Daniells, Gouwens, Herr, Holl, J. V. McKelvey, M. M. McKelvey, Pattengill, M. M. Roberts, P. G. Robinson, E. R. Smith, H. F. Smith, Snedecor, J. S. Turner.

CEDAR Falls. Daugherty, Kearney, Wester.

CEDAR RApids. Coffin.

DECORAH. Strom.

DES Moines. Hunter, P. C. Irwiu, Kennon, McCankie, MacKinnon, Nollen.

DUBuQue. Theobald. 
Fayette. Deming.

GrinNell. McClenon, Merriman, Rusk.

INDIANOLA. C. W. Emmons.

Iowa City. R. P. Baker, Chittenden, Conkwright, McCoy, J. F. Reilly,

H. L. Rietz, L. E. Ward, R. Woods, Wylie.

LE Mars. Blue.

Mount Vernon. McGaw, Moots.

Sioux City. Graber, Gwinn.

\section{KANSAS}

Atchinson. Pretz.

Baldwin City. Garrett.

Hays. Colyer.

Lawrence. Ashton, W. Babcock, F. L. Black, H. E. Jordan, U. G. Mitchell, G. W. Smith, Stouffer, J. J. Wheeler.

LINDSBORG. Marm.

Manhattan. Hyde, Mossman, Remick.

NEWTON. Richert.

Pittsburgh. Shirk.

SterLING. T. Bell.

TOPEKA. Harshbarger.

WichitA. Bernstorf, Longenecker, McShane, Mendenhall.

\section{KENTUCKY.}

Bellevue. Watts.

Glasgow. Hargrove.

LeXINGTon. Boyd, J. M. Davis, Downing, Latimer, Le Stourgeon.

LOUISviLle. Bullitt.

MURRay. Carman.

\section{LOUISIANA}

Baton Rouge. Sanders, Herman L. Smith, W. P. Webber.

NATCHITOChes. Maddox.

New Orleans. H. E. Buchanan, Cope, A. M. Howe, Lapeyre, Many, Spencer.

Pineville. C. D. Smith.

SHREVEPORT. Maizlish.

\section{MAINE}

Brunswick. E. S. Hammond, W. A. Moody.

LEWISTON. Ramsdell.

ORONO. Bryan.

WAterville. Ashcraft.

\section{MARYLAND}

Aberdeen. Dederick.

Annapolis. Bramble, Capron, Clements, Dillingham, Kells, Rawlins, Robert, Root, J. B. Scarborough, L. T. Wilson.
BAltimore. Bacon, Blumenthal, Cary

A. Cohen, Hagler, F. P. Lewis, F.

Morley, E. B. Morrow, Murnaghan,

L. J. Reed, L. B. Robinson, Sutton, Torrey, Zariski.

Cheltenham. Hartwell.

College Park. Dantzig.

WestMinster. McDaniel.

\section{Massachusetts}

Amherst. B. L. Brown, C. W. Cobb, Esty, Olds, C. S. Porter, Sprague.

BelmoNt. C. W. Franklin, S. $H$. Kimball.

Boston. Abbott, Bruce, Curry, Dysart, Elden, H. A. Garabedian, Gould, Grout, Hale, Hamilton, Hosmer, L. H. Howe, Kee, Mode, Moultrop, Norwood, Quigley, Rines, E. M. Thomas.

BROOKLINE. A. L. Miller.

Cambridge. L. B. Andrews, F. H. Bailey, Bartlett, Beatley, Birkhoff, Brinkmann, A. B. Brown, Byerly, Chafee, Clifford, Coolidge, P. Franklin, C. E. Fuller, Goodwin, W. C. Graustein, Hedlund, Hovgaard, $\dot{H}$. J. Hughes, Huntington, O. D. Kellogg, Kennelly, Keyes, Littauer, T. Lyman, C. L. E. Moore, M. Morse, Osgood, J. K. Peterson, H. B. Phillips, Poritsky, L. H. Rice, Rutledge, Shapley, Slotnick, M. H. Stone, Struik, Swain, Tyler, Vallarta, J. L. Walsh, Warner, Wexler, Wiener, Wildes, E. B. Wilson, E. W. Wilson, F. S. Woods, Zeldin.

Chestnut Hill. Lynch, McCormick, McHugh.

DORCHESTER. Sheffer.

Holyoke. Moriarty.

IPswich. Kinsman.

LyNN. G. W. Evans.

NAtick. R. Willis.

NEwTon. Schroeder.

Northampton. S. R. Benedict, Rambo, R. G. Wood.

Pittsfield. Bewley, Boyajian, Johannesen, Washburne, Wetherill.

SANDwICH. Bright.

South HADley. Anderton, Doak, Litzinger, E. N. Martin, S. E. Smith.

Swampscotr. Thomson.

Tufts College. Cheney, Ransom, F. G. Wren.

Wellesley. Comegys, L. P. Copeland, M. C. Graustein, H. A. Merrill, Pendleton, C. E. Smith, M. F. Smith, M. E. Stark, Vivian, A. H. Wheeler, Mabel M. Young.

West Lynn. Petrovsky, Summers.

Williamstown. Agard, Dorwart, J. G. Hardy, D. E. Richmond, V. H. Wells.

WINTHROP. Marden. 
Worcester. Gay, R. K. Morley, Patton, C. M. Rice, H. Rice, Hartley L. Smith, Story, Taber, F. B. Williams.

\section{MichigaN}

Alma. Clack.

ANN ARBor. Anning, Beale, Bradshaw, Coe, L. W. Cohen, C. C. Craig, Denton, Dushnik, P. Field, W. B. Ford, J. W. Glover, J. D. Grant, Grennan, Hildebrandt, L. A. Hopkins, Karpinski, Kazarinoff, C. E. Love, Markley, Nyswander, O. J. Peterson, Rainich, Rouse, Running, Shohat, R. L. Wilder, Ziwet.

Detroit. B. E. Allen, Frumveller, Ladue, A. L. Nelson.

East Lansing. L. C. Emmons, V. G. Grove, Heineman, W. S. Kimball, Olson, Plant, Powell, Speeker.

Fenton. Tryon.

Kalamazoo. H. Blair. Walton.

MARQUETTE. Spooner.

YPsilanti. Erikson, E. A. Lyman.

\section{MinNesota}

Minneapolis. W. O. Beal, Brink, Brooke, Bussey, Carlson, Dalaker, Earl, Gibbens, W. L. Hart, D. Jackson, Jensen, Mickelson, Risselman, Rojansky, Shumway, Underhill, Wilcox.

NoRTHField. M. B. White.

Rochester. W. I. Foster.

St Paul. W. H. Taylor, F. Wood.

Winona. Bogard.

\section{MISSISSIPPI \\ Agricultural College. B. M. Walker. \\ UNIVERSITY. Hume, Wunder.}

\section{MissourI}

Cameron. Marquis.

Canton. B. Ingold.

Carthage. G. E. Robinson.

Columbia. N. L. Anderson, H. Betz, Calloway, G. Cramer, Defoe, Haynes, L. Ingold, Jaeger, W. B. Smith, Spalding, Wahlin, Westfall, Wyant.

Kansas City. Luby, Melcher, R. M. Webb.

KIRKWOOD. Ammerman.

LIBERTY. Fleet.

MAYSVILle. Saunders.

Parkville. R. A. Wells.

Rolla. Hinsch.

St. Lours. L. Andrews, B u ri a n, Dunkel, Gerst, G. Graham, G. O. James, E. W. King, Lichter, Muehlman, C. E. Nelson, Osborn, Pennell,
Rafferty, Rider, Roever, Short, E.

Stephens, D. Wood, J. M. Young.

SPRINGFIELd. Finkel, Pummill.

WARRENSBURG. J. H. Scarborough.

\section{Montana}

Boseman. Hurst.

Helena. Rooney.

Missoula. Carey, Lennes, A. S. Merrill.

\section{Nebraska}

Lincoln. Brenke, C. C. Camp, Candy, Collins, Congdon, Doole, Engberg, Gaba, Gossard, Harper, Marvin, Maslow, Pierce, Runge, Sherer.

\section{Nevada}

Reno. C. Haseman, Searcy.

\section{NeW HAMPSHIRE}

Concord. G. M. Conwell.

DurHaM. Collignon, Slobin, Solt.

Hanover. Beetle, Bill, B. H. Brown, E. P. Brown, Forsyth, Haskins, Mathewson, Morgan, Perkins, J. M. Poor, Silverman, C. E. Wilder, J. W. Young.

\section{New Jersey}

Bayonne. Heimberger, W. A. Jones. BLOOMFIELD. Roman.

Convent Station. M. I. Johnson.

EAst Orange. Edison, Stanwick.

FraNkLIN Boro. Tillson.

Hoboken. L. E. Armstrong, H. N. Davis, Deimel.

LAWRENCEVILLE. Durell.

LEONIA. Gafafer.

Metuchen. Kennedy.

MONTClAIR. Mallory.

Newark. Brush, Conkling, Fitzgerald, Howell, Kineke, Lundgren, E. E. Rhodes, Skolnik, Sosnow, J. S. Thompson, Widmark.

New BRunswick. Brasefield, Hall, Huber, Meder, R. Morris, C. A. Nelson, Schoonmaker, Starke.

Ocean City. Groat.

Plainfield. Kircher.

Princeton. E. P. Adams, A. A Albert, Alexander, T. Bennett, Briggs, J. A. Clark, Cramlet, Derby, Eisenhart, Fine, D. A. Flanders, Flexner, A. L. Foster, W. Gillespie, Hille, Knebelman, Lefshetz, MacIn: nes, Singer, T. Y. Thomas, Veblen, Wedderburn, Willson.

RUTHERFORD. McMackin,

SOMERville. Moyle. 
Summit. H. E. Webb.

Trenton. L. L. Jackson.

\section{New Mexico}

Albuquerque. Barnhart.

LAS VEgAS. Rodgers.

State College. D. S. Robbins.

\section{NEW YoRK}

Albany. Birchenough, DoBell, Glasgow, Metzler, Stokes.

Alfred. Seidlin, Starr.

AnNandale-on-Hudson. Phalen.

AURORA. E. T. Carroll, Hollcroft.

Bellport. C. M. Walsh.

Binghamton. Patten.

Buffalo. A. H. Copeland, Sherk.

Canton. R. D. Ford.

Clinton. H. S. Brown, Carruth, Ferry, Fitch, Patterson.

Elmira. Suffa, Wright.

Geneva. W. H. Durfee, W. P. Durfee, Hubbs.

HAMILTON. Aude, A. W. Smith.

ItHACA. Agnew, Beisel, Boothroyd, Carver, C. F. Craig, D. C. Gillespie, Hadlock, Hurwitz, J. I. Hutchinson, Karapetoff, B. F. Kimball, Lowenstein, E. A. Miller, E. I. Moody, Paradiso, Ranum, Roos, Schug, Sharpe, V. Snyder, Swingle, Tanner, Torrance, Trevor, Willcox, F. G. Williams.

MARGARETVILLE. G. W. Walker.

New Rochelle. K. E. O'Brien, O'Callaghan.

NEw YORK. J. Allen, J. Alsberg, R. L. Anderson, R. G. Archibald, J. W. Arnold, Bassford, Belcher, Berger, Berkeley, Berman, William Johnston Berry, J. E. Black, V. Blair, Blake, Bowden, Breckenridge, R. W. Burgess, G. A. Campbell, R. V. Carpenter, Carrié, Carson, Castle, Chapell, J. D. Craig, Darkow, Dehn, Demos, Doermann, Douglas, Eckersley, T. W. Edmondson, Ellis, A. A. Evans, Fales, Farnum, Feld, Fenn, Fiske, Fite, Flinn, Foley, R. M. Foster, Frankel, F. Franklin, Frey, Fry, Gallatin, Gibbs, Gill, Gourin, P. H. Graham, W. J. Graham, Gronwall, C. C. Grove, Guggenbühl, Halbert, Hansell, I. Harter, Hartmann, Hausle, Hawkes, Hebbert, R. Henderson, L. S. Hill, Himwich, Hof mann, Hoyt, Hutcheson, R. L. Jackson, Jacobus, Joffe, R. A. Johnson, P. C. Jones, Kaplan, Kasner, Kenny, Kerr, Keyser, Kholodovsky, Koopman, Kopf, J. P. Kormes, M. Kormes, Korzybski, Kramer, Kunte, Langman, N. E. Lewis, Lieb, Lieber,
Linehan, Locke, Lotka, MacColl, MacNeish, M. Mason, S. P. Mead, Meyer, Middleton, Milligan, Mirick, Moir, Molina, Mullins, Murphy, F. H. Murray, Newman, Northcott, Oglesby, Otten, Paaswell, Packer, Pedersen, Pfeiffer, Plimpton, Pooler, C. L. Poor, Post, A. D. Pratt, A. G. Pratt, Preston, Pupin, R. G. Putnam, Raudenbush, Reddick, M. S. Rees, F. G. Reynolds, Ritt, Roosevelt, Ruger, Saurel, Schub, Schuyler, P. D. Schwartz, Seely, Serghiesco, Sharp, Shewhart, Siceloff, L. G. Simon, Simons, D. E. Smith, F. M. Smith, P. A. Smith, R. F. Smith, Snook, F. R. Stark, Strong, Tanzola, J.E. Thompson, Thorndyke, Thorne, Upton, Vedova, Vreeland, E. Walker, H. Webster, Wechsler, Weeks, Weisner, Wetzel, D. E. Whitford, E. E. Whitford, Whitney, E. C. Williams, Yanosik, Margaret M. Young, Zobel.

Niagara Falls. W. L. Bliss.

OnEonta. A. M. Curtis.

Parish. E. Church.

Potsdam. J. E. Rowe.

PoughkeEPsie. Cowley, L. D. Cummings, G. Smith, M. E. Wells, H. S. White.

Rochester. Altman, Atwater, W. Betz, Bishop, Bunnell, Dye, Frederick, Gale, L. A. Jones, Long, Silberstein, Van de Walle, Watkeys, R. A. Whelan.

St. Bonaventure. A. O'Brien.

SchenECTADY. Alger, Berg, Buller, Burkett, Dushman, Garis, Harms, M. L. Henderson, Herod, Howard, Hussey, E. E. Johnson, W. C. Johnson, E. W. Kellogg, A. L. Kimball, Langmuir, Leibing, Lorraine, McLenegan, Male, D. S.. Morse, Mott-Smith, Newkirk, Park, Prince, C. W. Rice, E. L. Robinson, Skeats, J. C. Smith, J. J. Smith, A. D. Snyder, Steinert, H. D. Taylor, Terry, Vedder, Wieseman.

Syracuse. Biklé, A. D. Campbell, I. S. Carroll, Decker, Lindsey, E. D. Roe, J. R. Roe, M. J. Sperry, Wolverton.

Tonawanda. Steck.

Troy. Crockett, McGiffert.

West Point. C. P. Echols.

\section{North Carolina}

Chapel Hill. Browne, Cain, A. Henderson, Lasley, Mackie.

DURHAM. Clarkson, W. W. Elliott, W. W. Rankin, Robison.

Elon College. Amick. 
Greensboro. Pegram, Ragsdale, Watt.

KitTrell. Thornton.

LOUISBURG. Bizzell.

Murfreesboro. Caldwell.

\section{North Dakota}

FARGo. Grimes, I. W. Smith.

GRAND FoRKS. Hitchcock.

\section{OHIO}

Akron. H. A. Bender, J. L. Jones. Athens. Borger, F. W. Reed.

BARBERTON. Langvand.

Bluffton. Hirschler.

Bowling Green. Overman.

Cincinnati. Barnett, C. M. Biscay, Jr., C. M. Biscay, Sr., Brand, Carrington, Chenoweth, Farnau, C. A. Garabedian, Hancock, E. E. Hardcastle, Hundley, Kersten, Kindle, Koeppe, Lehenbauer, Lubin, Metz, H. L. Miller, C. N. Moore, Mullings, C. E. Rhodes, Ryan, Sibert, Stillwell, Wilczewski, Winston, Yowell.

Cleveland. Boyce, Burington, Burwell, Feinler, Focke, Jonah, Kraft, J. E. Merrill, D. C. Miller, M. Morris, Musselman, Nassau, Palmié, W. G. Simon.

Columbus. C. L. Arnold, Bareis, Blumberg, Harmount, Kuhn, MacDuffee, Manson, Michal, C. C. Morris, T. S. Peterson, Rasor, J. C. Rietz, J. H. Weaver.

DAyton. Hartwick, Midgley.

Delaware. Crane, Newlin, Rowland.

Galion. McBerty.

Gambier. R. B. Allen.

GRANVILLE. Wiley.

HiRam. Clarke.

KEnT. Manchester.

NAPOLEON. E. M. Weaver.

NoRwOod. Wishard.

Oberlin. Cairns, F. E. Carr, M. M. Johnson, Sinclair, Yeaton.

OXFORD. W. E. Anderson, Pollard, Spenceley, Tappan.

Painesville. A. D. Lewis.

SPRINGFIELd. Tripp.

Westerville. B. C. Glover.

WILBERFORCE. Tinner.

WiLMINGTON. Spinks.

Wooster. C. O. Williamson, Yanney.

Yellow SpRINGs. Dwyer.

\section{OKLAHOMA}

ENID. B. V. Hill.

Norman. Brixey, Court, Hassler, McFarland, Meacham, Raynor, S. W. Reaves.

StillWATER. R. L. Flanders, Gundersen.

TULSA. Galbraith, Rutherford.

\section{OREGON}

Albany. Butler.

Corvallis. Kent.

Eugene. D. R. Davis, De Cou, Hicks, McAlister.

McMinnville. Ramsey.

PoRTLAND. Biggerstaff, Copenhaver, Donahue, Griffin.

\section{Pennsylvania}

Allentown. Callahan, Deck.

ANNville. Grimm, P. S. Wagner.

BALA-CyNwyd. Gummere.

BeAver Falls. Cleland.

Bethlehem. Fort, Hammatt, Koehler, Lamson, McDonough, Ogburn, Rau, Riddle, Smail, Stocker, Trjitzinsky, Weida.

BROOKLINE. Lamond.

Bryn Mawr. Lehr, Pepper, Pixley, Scott, A. P. Wheeler, Widder.

CARLISLe. Landis.

Charleroi. Shriver.

Collegeville. Clawson.

EAston. Fithian, Hatch, W. M. Smith.

Ellwood City. Brownstein.

Fullerton. Cassidy.

Gettysburg. Arms, Clutz.

GreENVILLE. Noecker.

HARRISBURG. Whited.

HAVERFORD. Reid, A. H. Wilson.

Immaculata. Mary Columba, Mary St. Bernard.

LANCASTER. Witmer.

LEWISBURG. Lindemann, MacCreadie.

MEADVILlE. Akers, W. J. Wagner.

Millersville. Seiverling.

New Wilmington. McCain.

Philadelphia. M. Allen, Babb, F. W. Beal, Bristol, Brumbaugh, Bryant, Caris, Chambers, Crawley, J. E. Davis, Eshleman, H. B. Evans, Gleason, Glenn, H. S. Grant, Hallett, M. L. Johnson, Kline, Knobelauch, Linton, H. H. Mitchell, Partridge, Perrin, M. Reilly, Rittenhouse, Rorer, Rutt, Safford, A. F. Schwartz, Schwatt, Shoemaker, Shugert, M. B. Snyder, Stevens, J. M. Thomas, Weinstein, F. H. M. Williams, Willows, Wolff, W. Wood, Zippin.

Pittsburgh. Burns, H. D. Curtis, Foraker, R. P. Johnson, Morehead, Neelley, Riggs, Rosenbach, Schnurmann, Slepian, Staniland, Swartzel, J. S. Taylor,

READING. Steinmetz.

Saylorsburg. W. M. Bond.

Scranton. Mary Bertrand (Walton). SHIPPENSBURG. J. S. Grove.

State College. T. Cohen, Frink, Gravatt, Johnston, McCarthy, 
Mears, F. W. Owens, H. B. Owens, Rosinger, Rupp, Stecker.

SwarthmoRe. Dresden, Kern, Marriott, J. A. Miller.

TRAPPE. Hiltebeitel.

Washington. Atchison, Shaub.

WAyNE. Maddison.

\section{Porto Rico}

Mayaguez. Morales, Sanchez-Diaz. Rio Piedras. Kuschke.

San Juan. Horne.

\section{RHODE ISLAND}

NeWPORT. R. S. Kimball.

Providence. C. R. Adams, R. B. Adams, R. C. Archibald, A. A. Bennett, Carlen, Chace, Currier, Gilman, Hickson, D. H. Lehmer, Lepeshkin, H. P. Manning, Randall, R. G. D. Richardson, A. H. Smith, Suesman, Tamarkin, Thurston.

\section{South Carolina}

Columbia. Coleman, J. B. Jackson. Greenville. Bowen, Earle, R. B. Wood.

Hartsville. C. M. Reaves.

OrANGEBURG. Nix.

Rock Hrll. A. A. Grant, Watkins.

\section{South Dakota}

Aberdeen. Hobart.

BrookIngs. I. L. Miller.

Rapid City. Bowles.

Vermillion. H. I. Lane, McKinney.

YANKTON. Faught.

\section{Tennessee}

JACKson. Carr.

KNOXVILle. J. D. Bond.

MEMPHIs. Hartley, McQueen.

Nashville. Hawks, Hyden, Miser, F. L. Wren.

\section{Texas}

Abilene. Burnam, Tate

Austin. Batchelder, H. Y. Benedict, Calhoun, Decherd, Dodd, Dorroh, Ettlinger, Holmes, Horton, Keller, Lowber, Lubben, R. L. Moore, Muller, M. B. Porter, C. D. Rice, J. H. Roberts, Vandiver, G. T. Whyburn.

BOERNE. Hathaway.

College Station. F. Ayres, Halperin, W. A. Rees.

Dallas. Hosford, E. H. Jones, Montgomery, Reinsch.

Houston. Bray, Dean, N. Edmonson, G. C. Evans, L. R. Ford, Gergen, R. N. Haskell, Hickey, Lovett, E. R. C.
Miles, W. L. Porter, Ricker, H. A. Wilson.

JACKSON. Walden.

LuBBock. Michie, L. V. Robinson, Sparks, Stafford, Wait.

SAN ANTonio. Udinski.

Sherman. May.

Waco. J. B. Johnson.

WaXahachie. Newton.

\section{UTAH}

Salt Lake City. J. L. Gibson, Pehrson, Unseld.

\section{VERMONT}

Burlington. Bullard, Fraleigh, Swift, E. Thomas.

MiddlebuRy. L. R. Perkins.

MONTPELIER. Blackmore, Hobson, Holt, H. H. Jackson, C. E. Moulton.

\section{VIRGINIA}

Ashland. T. McN. Simpson.

BLACKSBURG. Brodie, O'Shaughnessy, J. E. Williams.

BRIDGEWATER. Shull.

DAHLGREN. L. T. E. Thompson.

EMORY. J. S. Miller.

Hampton Institute. H. A. Perkins. HoLlins, Dickinson.

Lexington. Cocke, Paxton

LyNChBurg. E. M. Berry, A. Church, Larew, Pattillo.

RICHMOND. Amig, Gaines, E. J. Willis. Sweet Briar. Bower, Calkins, Morenus.

TownsEnd. Parsons.

UNIVERsITY. W. H. Echols, Linfield, Luck, J. M. Page, Sparrow, O. Stone.

WILliamsBURG. Stetson.

\section{WASHINGTON}

Pullman. E. C. Colpitts, Isaacs, Schelkunoff, Vatnsdal.

Seattle. C. R, Ballantine, J. P. Ballantine, A. F. Carpenter, Gavett, Jerbert, Langford, McFarlan, Magnusson, Moritz, Mullemeister, Neikirk, Winger, Zanstra.

TACOMA. Hanawalt.

Walla Walla. Bratton.

\section{WEST ViRgINIA}

Barboursville. Byrne.

Bethany. Cramblet.

Huntington. Hackney.

Institute. E. F. Cox

Morgantown. M. Buchanan, Colwell, H. A. Davis, Eiesland, H. A. Hoover, Mathews, C. N. Reynolds, B. M. Turner.

WheEling. Bagby. 


\section{Wisconsin}

Beloit. H. H. Conwell.

Madison. F. E. Allen, R. W. Babcock, G. N. Carmichael, H. V. Craig, Dancer, Dowling, H. P. Evans, Gilmore, W. W. Hart, Hartung, Hayden, Ingraham, Langer, March, Parkinson, Skinner, Slichter, Sokolnikoff, J. H. Taylor, Van Vleck, Vass, W. Weaver.

Matroon. Melchiors.

MILTON. F. Hopkins.
Mrlwaukee. Battig, Bear, E. R. Beckwith, L. H. Bunyan, Chapman, Clary, P. H. Evans, Knight, Laun, Pettit, Roth, Sperling.

Superior. C. W. Smith.

WaUkesha. Dancey.

West De Pere. De Cleene.

Williams Bay. Pogo.

Wisconsin RAPIDS. McMillan.

\section{Wyoming}

LARAMIE. Bellamy, Rechard.

\section{ARGENTINA}

Buenos Arres. Baidaff, Jaime, Posey.

AUSTRIA

Vienna. W. L. Ayres.

BRAZIL

Pernambuco. Riberio.

BRITISH EMPIRE

Ayr. Gray.

\section{Great Britain}

BANGor. Berwick.

BIRMINGHAM. Watson.

Cambridge. A. Berry, Larmor, Macaulay, H. W. Richmond.

CARDIFF. Livens.

DUNDEE. Steggall.

EDINBURGH. E. T. Whittaker.

Glasgow. G. A. Gibson.

Hull. Forder.

KInROss. J. Williamson.

LEEDS. W. P. Milne.

LIVERPool. B. M. Wilson.

London. B. B. Baker, Brill, Hilton, Western.

Manchester. Mordell.

OxFORD. Dixon, E. B. Elliott, G. H. Hardy, A. E. H. Love, Srivastava.

ShefFIELD. Daniell.

Stocksfield-on-Tyne. F. Hardcastle.

Swansea. A. R. Richardson.

\section{Australia}

Adelaide. Wilton.

Sydney. Carslaw.

\section{BURMA}

INSEIN. Aiyar.

Rangoon. W. B. Campbell.

\section{Canada}

Edmonton. J. W. Campbell, Cook, Sheldon.

Fredericton. C. C. Jones.

GrimsBy. Wolfenden.
KIngston. Gummer, Matheson, N. Miller.

LONDON. Kingston.

Montreal. Beaupré, L. V. King, MacLean, D. A. Murray, Pellétier, A. N. Shaw, Sullivan, W. L. G. Williams.

Ottawa. Glashan, McDonnell, R. M. Stewart.

Quebec. Pouliot, A. V. Richardson.

Regina. W. H. McEwen.

Saskatoon. Dines, Ling.

Toronto. Beatty, De Lury, Fields, Findlay, Gilchrist, Pounder, Rosebrugh, Stevenson, L. B. Stewart, Waddell, W. J. Webber.

VANCouver. D. Buchanan, F. S. Nowlan.

Victoria. Gage.

WINNIPEG. T. H. Milne, Riter, Warren, N. R. Wilson.

Wolfville. Jeffery.

INDIA

BAREILly. Bhattacharyya.

Calcutta. Prasad.

LAHORE. Sharma.

\section{IRELAND}

Cork. Conran

Dublin. C. H. Rowe, Synge.

New Zealand

Wellington. Sommerville, Weatherburn.

\section{South Africa}

RONDEBOSCHE. Muir.

Pexing. Konantz, Sun. Tientsin. Chang, Chiang. 


\section{DENMARK}

Copenhagen. Nörlund.

Carro. Boulad, Ince.

\section{EGYPT}

\section{FRANCE}

LILLE. Chapelon.

Paris. de Séguier.

STRASBOURG. Fréchet.

\section{GERMANY}

BERLIN. Thurow.

FreIBURG. Bolza.

Charlottenburg. Korn.

FrankFort A. M. Szasz.

GÖTTINGEN. W. V. Bond.

LEIPZIG. Ackermann-Teubner.

GREECE

Athens. Sakellariou, Zervos.

Budapest. C. Jordan.

\section{HUNGARY}

ITALY

Rome. Castellani, Levi-Civita, Volterra.

\section{JAPAN}

MIYAZAKI-Ken. Matsumura.

SAPPORO. Kawaguchi.

SENDAI. Hayashi, Kubota, Okada, Takasu.

Tokyo. Kuniyeda.

Yamagata. Yanagihara.

Yedajima. Mori.

Coahuila. García.

$$
\text { MEXICO }
$$

TAcubaya. Gallo.

Horten. Naess.

$$
\text { NORWAY }
$$

\section{PERU}

Lima. de Losada y Puga.

PHILIPPINE ISLANDS

Manila. Artiaga, Tan, Tienzo.

WARSAW. Dickstein.

$$
\text { POLAND }
$$

\section{RUSSIA}

LENINGRAd, Smouroff.

\section{TURKEY}

Constantinople. Sarafoglou.

VENEZUELA

Caracas. Arcaya, Nouel.

Maracaibo. Lugo. 